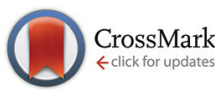

Cite this: Dalton Trans., 2016, 45 14058

Received 1st August 2016,

Accepted 18th August 2016

DOI: $10.1039 / c 6 d t 03047$ e

www.rsc.org/dalton

\section{Designed intramolecular blocking of the spin crossover of an Fe(II) complex $\dagger$}

\author{
C. Bartual-Murgui, ${ }^{\star a}$ S. Vela, ${ }^{\star b}$ O. Roubeau ${ }^{c}$ and G. Aromíta
}

A ligand derived from 1,3bpp (2-(pyrazol-1-yl)-6-(pyrazol-3-yl)pyridine) has been prepared to prove that the spin crossover (SCO) of an $\mathrm{Fe}$ (II) complex can be blocked by means of intramolecular interactions not related to the crystal field. Calculations show that the blocking is caused by the energy penalty incurred by the rotation of a phenyl ring, needed to avoid steric hindrance upon SCO.

The exploitation of spin crossover (SCO) offers an opportunity for the implementation of a molecular-based switching property at the nano-scale. ${ }^{1-3}$ The phenomenon is displayed by transition metals that exhibit two possible $d$ electron configurations, and a ligand field causing these to lie close in energy, so that they can be easily interconverted through external stimuli. The spin transition occurs along the changes to the structure, together with variations in physical properties such as colour, ${ }^{4}$ magnetic ${ }^{5-7}$ or electrical ${ }^{8}$ properties, mechanical responses, ${ }^{9}$ etc. The dynamics of spin transition, crucial for exploiting the switching behaviour, is governed by elastic interactions between active centres within the host crystal lattice. Thus, the presence of a dense network of intermolecular or covalent interactions often leads to cooperative SCO, which translates into abrupt transitions, sometimes accompanied by hysteresis. ${ }^{10,11}$ Therefore, great efforts are dedicated to unveil the subtle links between SCO and the associated changes to the crystal lattice. ${ }^{12-14}$ In this context, structural analyses indicate that complexes with the appropriate ligand field to undergo SCO may be kinetically trapped in the high spin (HS)

\footnotetext{
${ }^{a}$ Departament de Quimica Inorgànica i Orgànica and IN2UB, Universitat de Barcelona, Diagonal 645, 08028 Barcelona, Spain. E-mail: guillem.aromi@qi.ub.es ${ }^{b}$ Laboratoire de Chimie Quantique, Université de Strasbourg, 4 rue Blaise Pascal, F-67000 Strasbourg, France

'Instituto de Ciencia de Materiales de Aragón (ICMA), CSIC and Universidad de Zaragoza, Plaza San Francisco s/n, 50009 Zaragoza, Spain

$\dagger$ Electronic supplementary information (ESI) available: Ligand synthesis details, crystallographic tables and additional figures, PXRD patterns, TGA graphs, and additional magnetocaloric graphs. CCDC 1492563 and 1492564. For ESI and crystallographic data in CIF or other electronic format see DOI: 10.1039/ c6dt03047e
}

state if the transition requires excessive crystal lattice rearrangement energy, which is usually gauged by a very distorted coordination geometry. ${ }^{15,16}$ This was also demonstrated by means of $\mathrm{DFT}+U$ calculations in the solid state. ${ }^{17}$ Complementary to this, by studying the Fe(II) complexes of a family of indazolylpyridine ligands, it was proposed that the relative stability of the HS and low spin (LS) states may be strongly modulated by intra-ligand steric interactions. ${ }^{18}$ In a theoretical investigation, it was suggested that the true reason for the drastic differences within that series of compounds is indeed inter-ligand (while intra-molecular) steric factors. ${ }^{19}$ Along the same lines, following the study of a series of HS $\mathrm{Fe}(\mathrm{II}) / 1 \mathrm{bpp}$ derivatives, it was suggested that steric intramolecular interactions could be playing a role in blocking the SCO. ${ }^{20}$ With the aim of elucidating these factors, we have prepared and used a ligand that serves to demonstrate that a complex expected to show SCO can be completely trapped into the HS state by virtue of purely intra-molecular, non-covalent interactions and not due to a lattice effect. A thorough DFT analysis provides an elegant rationalization of this behaviour.

The new ligand Ph1,3bpp (2-(3-phenylpyrazol-1-yl)-6-(1Hpyrazol-3-yl)-pyridine; Scheme 1) was prepared in three steps, starting from the coupling of 3-phenylpyrazole with 2-acetyl-6bromopyridine, followed by the isolation of the corresponding propanone intermediate that results from the activation of the acetyl group with 1,1-dimethoxytrimethylamine, which upon ring-closure with hydrazine yields the desired product (ESI $\dagger$ ).
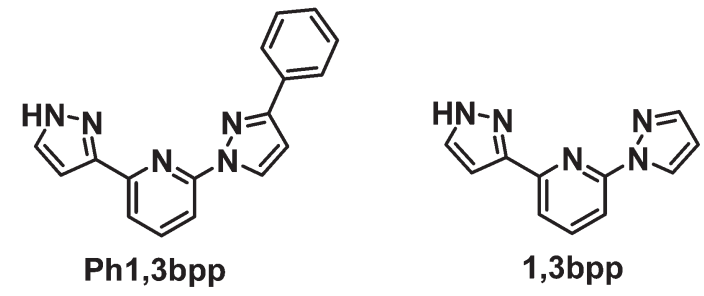

Scheme 1 Molecular structure of ligands Ph1,3bpp and 1,3bpp. 
The reaction of $\mathrm{Ph} 1,3 \mathrm{bpp}$ with $\mathrm{Fe}\left(\mathrm{ClO}_{4}\right)_{2} \cdot 6 \mathrm{H}_{2} \mathrm{O}$ in dry acetone produced the crystals of the compound $\left[\mathrm{Fe}(\mathrm{Ph} 1,3 \mathrm{bpp})_{2}\right]\left(\mathrm{ClO}_{4}\right)_{2} \cdot \mathrm{C}_{3} \mathrm{H}_{6} \mathrm{O}$ (1a) upon layering the initial mixture with $\mathrm{Et}_{2} \mathrm{O}$. Compound 1a crystallizes in the orthorhombic space group $P c a 2_{1}$. The asymmetric unit (Fig. S1†) contains two complete formula units, which are very similar but not crystallographically equivalent. The unit cell includes four asymmetric units. Crystallographic data and metric parameters can be found in the ESI. $\dagger$ The basic unit of 1a comprises a $\left[\mathrm{Fe}(\mathrm{Ph} 1,3 \mathrm{bpp})_{2}\right]^{2+}$ complex cation with the charge compensated by two $\mathrm{ClO}_{4}{ }^{-}$anions and one molecule of acetone of crystallization (Fig. 1). The complex consists of an $\mathrm{Fe}(\mathrm{II})$ centre coordinated by two tridentate Ph1,3bpp ligands in a mer fashion, completing a very distorted $\mathrm{FeN}_{6}$ coordination geometry. The average of the Fe-N bond distances is 2.16(4) $\AA$, for the two different Fe(II) ions, which shows that both of them remain in the $\mathrm{HS}$ state at $100 \mathrm{~K}$, here as a result of intra-molecular, inter-ligand interactions (see below). The severe distortion of the metal environment from the ideal octahedron as gauged by the parameters $\Sigma$ and $\Theta^{7}$ (here with average $\Sigma$ and $\Theta$ values of $168.8(9)$ and $521(2)$, respectively) is also witness to the HS state. ${ }^{21}$ This strong distortion could also cause the HS of this compound to be kinetically trapped.

The conformation of the Ph1,3bpp ligands within the complex must be emphasized; the phenyl substituents are in all the cases twisted with respect to their adjacent pyrazolyl rings with the angles of $47.71 / 46.56^{\circ}$ and $49.24 / 47.75^{\circ}$, and establish intramolecular $\pi \cdots \pi$ interactions with their opposite $1,3 \mathrm{bpp}$ fragments in the complex (Fig. S2 $\dagger$ ). These $\pi \cdots \pi$ interactions are slightly different in their strength and position. Their strength may be gauged by the distance between the $\mathrm{Ph}$ ring centroid and the idealized 1,3bpp plane (here $3.412 \AA$ in average). The above two factors likely play a role in the pronounced mutual rotation of both $1,3 \mathrm{bpp}$ moieties away from orthogonality (angles between the idealized planes of 69.79 and $71.53^{\circ}$ ). The $\mathrm{N}-\mathrm{H}$ groups of the complex interact through strong hydrogen bonds with the acetone molecule and one

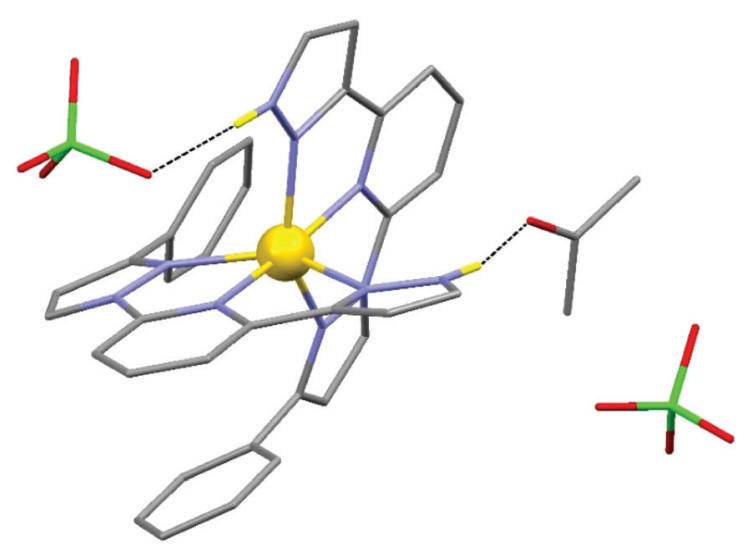

Fig. 1 Molecular representation of one of the formula units in [Fe(Ph1,3bpp $\left.)_{2}\right]\left(\mathrm{ClO}_{4}\right)_{2} \cdot \mathrm{C}_{3} \mathrm{H}_{6} \mathrm{O}$ (1a). Fe is the yellow ball, $\mathrm{C}$ is grey, $\mathrm{N}$ is purple, $\mathrm{O}$ is red, $\mathrm{Cl}$ is green. Only $\mathrm{H}$ atoms of the $\mathrm{N}-\mathrm{H}$ groups are shown (in yellow). Dashed lines are $\mathrm{H}$-bonds. perchlorate anion, respectively. The other $\mathrm{ClO}_{4}{ }^{-}$group in the formula is subjected to weaker interactions within the lattice. This happens for both complexes of the asymmetric unit. The complexes are disposed in sheets that contain both possible enantiomers, where they interact with each other only via $\mathrm{C}-\mathrm{H} \cdots \pi$ interactions (Fig. S3†). Thus, the organization within these sheets does not follow the compact network of $\mathrm{C}-\mathrm{H} \cdots \pi$ and $\pi \cdots \pi$ contacts known as the "terpy embrace" usually encountered in complexes with bpp type ligands. ${ }^{22}$ Acetone and $\mathrm{ClO}_{4}{ }^{-}$anions are located in between the sheets of complexes, yielding a lattice that alternates essentially hydrophobic with rather hydrophilic layers (Fig. S4 $\dagger$ ). The sheets of metal complexes feature two significantly different separations (amounting to 9.368 and $12.190 \AA$, respectively). Half of the $\mathrm{ClO}_{4}{ }^{-}$anions in the compound and all the molecules of acetone are located in the space between the layers with the largest separation, whereas the remaining anions lie in the other inter-sheet domain (Fig. 2). In contact with air, compound 1a exchanges all its acetone content with half equivalent of water molecules, in a single-crystal-to-single-crystal (SCSC) manner. This transformation does not lead to any apparent changes while single-crystal X-ray diffraction allows the determination of the molecular structure of the new product, [Fe(Ph1,3bpp $\left.)_{2}\right]$ $\left(\mathrm{ClO}_{4}\right)_{2} \cdot 0.5 \mathrm{H}_{2} \mathrm{O}$ (1b). Thermogravimetric analyses (TGA) also confirm the process (Fig. S5 $\dagger$ ). This system joins a growing family of bpp-based $\mathrm{Fe}(\mathrm{II})$ complexes that experience SCSC solvent exchange processes allowing the use of SCXRD to study the initial and the final forms, ${ }^{23-26}$ and sometimes even intermediate phases. ${ }^{27}$ Compound $\mathbf{1 b}$ is found in the orthorhombic space group $\mathrm{Pna}_{1}$, its asymmetric unit (Fig. S6†) comprising two inequivalent $\left[\mathrm{Fe}(\mathrm{Ph} 1,3 \mathrm{bpp})_{2}\right]^{2+}$ complexes, four $\mathrm{ClO}_{4}{ }^{-}$ions and one molecule of water. The latter occupies a position equivalent to one of the acetone molecules in 1a, also forming a H-bond with one $\mathrm{N}-\mathrm{H}$ group. The remaining $\mathrm{N}-\mathrm{H}$ groups that were interacting with the other acetone in 1a are now the donors of a $\mathrm{H}$-bond with one $\mathrm{ClO}_{4}{ }^{-}$ion. Therefore, after the molecular movements occurring during the $\mathbf{1 a} \rightarrow \mathbf{1 b}$ transformation, one third of the $\mathrm{ClO}_{4}{ }^{-}$groups are now involved in $\mathrm{N}-\mathrm{H} \cdots \mathrm{O}\left(\mathrm{ClO}_{3}\right)^{-}$interactions. This molecular exchange does not lead to significant changes in the conformation and structural parameters of the $\left[\mathrm{Fe}(\mathrm{Ph} 1,3 \mathrm{bpp})_{2}\right]^{2+}$ moieties (see ESI $\dagger$ ), which also remain, as expected, in the HS state at $100 \mathrm{~K}$ (average Fe-N distances of 2.166(11) and 2.165(11) $\AA$ ). The $\mathrm{Ph} / \mathrm{pz}$ torsion angles are now $45.35 / 47.27^{\circ}$ and $47.97 / 46.35^{\circ}$ and the average Ph-centroid to the idealized 1,3bpp plane distance is $3.442 \AA$ A. Likewise, the layered organization of the complex cations within the crystal lattice does not vary. The sheets are separated by the perchlorate groups, featuring two types of separations (now of 9.422 and $10.551 \AA$, respectively). The larger interlayer domain also includes the molecules of water (Fig. 2). It is thus clear that the space located in between the largest inter-layer separation mediates the diffusion of molecules transiting in and out of the lattice. The exchange process causes a $9 \%$ contraction of the lattice.

Contrary to most SCSC small-molecule exchanges reported for the family of bpp/Fe(II) complexes, ${ }^{23-26}$ the $\mathbf{1 a} \rightarrow \mathbf{1 b}$ transformation does not produce a spin switching (at least down to 

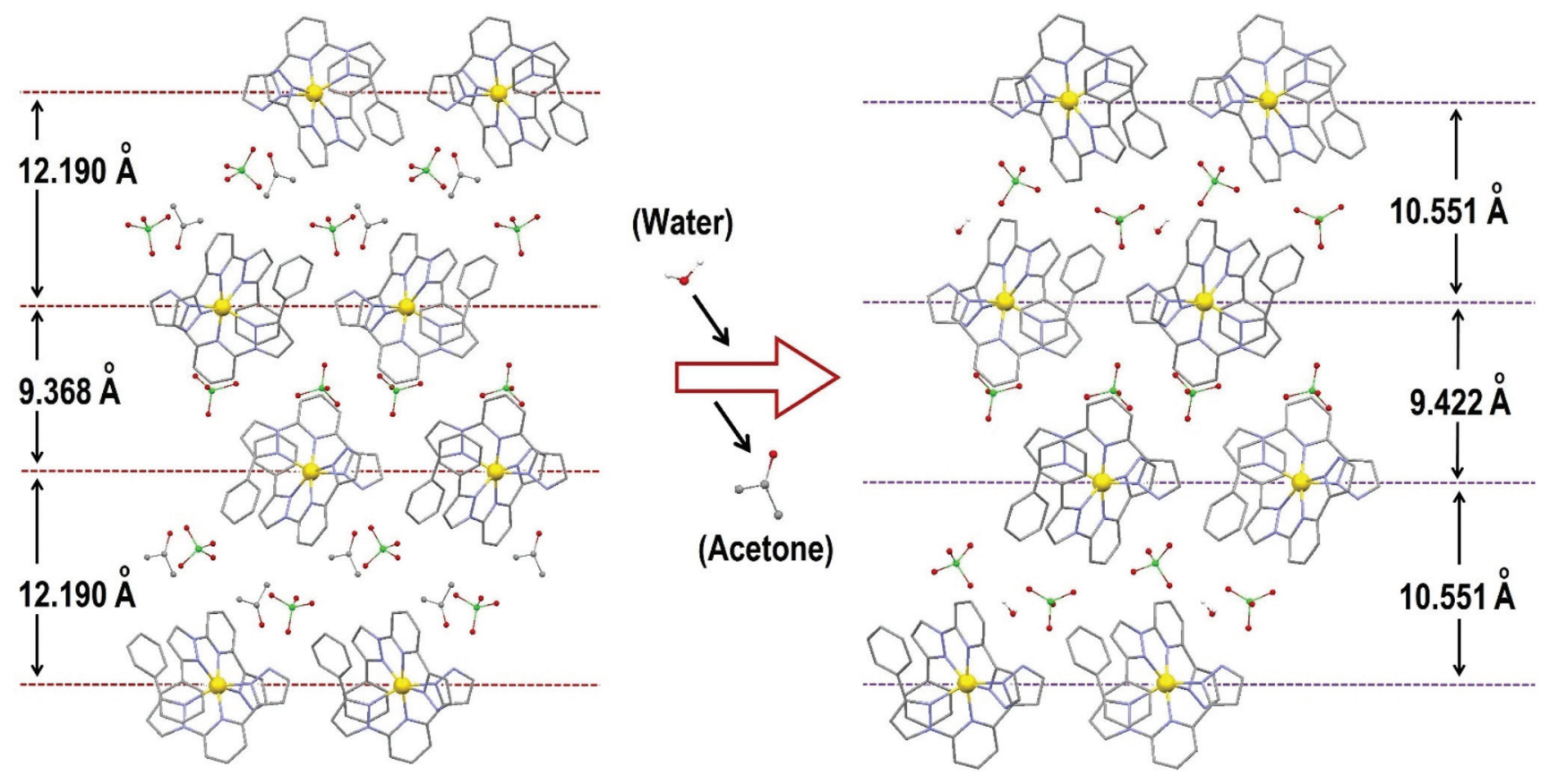

Fig. 2 View of the lattices of $1 \mathrm{a}$ (left) and $1 \mathrm{~b}$ (right) down the crystallographic $b$ axis, emphasizing the solvent exchange that takes one into the other and the two interlayer separations hosting or not the migrating molecules. Code: balls, $\mathrm{Fe}$; red, $\mathrm{O} ; \mathrm{green}, \mathrm{Cl} ; \mathrm{grey}, \mathrm{C}$. Hydrogens are not shown.

$100 \mathrm{~K})$ from the HS state. This was corroborated by means of magnetic susceptibility measurements down to $5 \mathrm{~K}$ (Fig. S7 $\dagger$ ). Thus, under a constant magnetic field of $0.1 \mathrm{~T}$, compounds 1a and $\mathbf{1 b}$ exhibit values of the $\chi_{\mathrm{M}} T$ product $\left(\chi_{\mathrm{M}}\right.$ is molar paramagnetic susceptibility) of 4.04 and $3.94 \mathrm{~cm}^{3} \mathrm{~K} \mathrm{~mol}^{-1}$ at 350 and $301 \mathrm{~K}$, respectively, consistent with both systems being completely in the HS state $(S=2)$. The Curie Law behaviour holds in both cases down to below $50 \mathrm{~K}$, from where the $\chi_{\mathrm{M}} T$ vs. $T$ curve plots exhibit the sharp expected decline resulting from zero field splitting effects on HS Fe(II).

The ligand Ph1,3bpp was designed to prove that intramolecular interactions could be exploited to block the SCO and trap a complex in the HS state. The magnetic response of 1a and the fact that the exchange of acetone/water does not affect this response point in this direction. For comparison, the related complex $\left[\mathrm{Fe}(1,3 \mathrm{bpp})_{2}\right]\left(\mathrm{ClO}_{4}\right)_{2}(2){ }^{28}$ which features the same core as $\mathbf{1 a}$ and $\mathbf{1} \mathbf{b}$ without the Ph substituent, exhibits a complete SCO close to $275 \mathrm{~K}$ (Fig. S7†), which also supports this hypothesis. An elegant explanation for the SCO blocking of $\mathbf{1 a}$ and $\mathbf{1 b}$ was given by means of computational tools. Thus, the optimized geometries associated with the HS and hypothetical LS states of the complex $\left[\mathrm{Fe}(\mathrm{Ph} 1,3 \mathrm{bpp})_{2}\right]^{2+}(\mathbf{1})$ under gas-phase conditions were determined, starting from the crystal coordinates (Fig. S8 $\dagger$ ). The calculated HS structure $\left(\mathbf{1}_{\mathrm{HS}}\right)$ is very similar to the experimental one, reproducing the Fe-N distances, and most notably, the distortion of the coordination geometry (average Fe-N distance, $2.191 \AA$ A; $\Sigma=165.04$; $\Theta=530)$. The structure obtained for the hypothetical LS state $\left(\mathbf{1}_{\mathrm{LS}}\right)$ features the expected compression of the $\mathrm{FeN}_{6}$ core (average Fe-N value, $1.963 \AA$ ), which also becomes closer to an ideal octahedron $(\Sigma=99.26 ; \Theta=308)$. Interestingly, in both cases, the phenyl substituents of the ligands are twisted with respect to their respective $1,3 \mathrm{bpp}$ planes (Fig. $\mathrm{S} 8 \dagger$ ), as also observed experimentally. This could be ascribed to a tendency for establishing favourable inter-ligand $\pi \cdots \pi$ interactions (see below). The calculated energies of both forms predict higher electronic enthalpy for $\mathbf{1}_{\mathrm{HS}}\left(\Delta H_{\text {elec }}=-1.7 \mathrm{~kJ} \mathrm{~mol}^{-1}\right)$, which would indeed preclude the thermal SCO, since the latter is an entropy driven process. In order to compare with 2 , single point energy evaluations were performed on $\mathbf{1}_{\mathrm{HS}}$ and $\mathbf{1}_{\mathrm{LS}}$ without the phenyl substituents. This results in a dramatic inversion of the relative HS vs. LS electronic enthalpies, now giving $\Delta H_{\text {elec }}=+10.8 \mathrm{~kJ} \mathrm{~mol}^{-1}$ and restoring the thermodynamic stability of the LS state, consistent with the experimental results. ${ }^{28}$ Both sets of calculations confirm that the blocking of the SCO in $\mathbf{1}$ is entirely related to the phenyl groups. The influence of the intramolecular $\pi \cdots \pi$ interaction was examined using several models. It was found that this interaction causes a difference in stability of only -0.1 to $-0.5 \mathrm{~kJ} \mathrm{~mol}^{-1}$ in favor of the HS state. Such contribution is nearly two orders of magnitude smaller than the overall effect of ca. $12 \mathrm{~kJ} \mathrm{~mol}^{-1}$ caused by the $\mathrm{Ph}$ rings (by adding $\Delta H_{\text {elec }}$ in systems 1 and 2, i.e. -1.7 and $10.8 \mathrm{~kJ} \mathrm{~mol}^{-1}$ ). Another difference between $\mathbf{1}_{\mathrm{HS}}$ and $\mathbf{1}_{\mathrm{LS}}$, also related to the phenyl substituent of Ph1,3bpp, is the torsion angle between this substituent and their carrier pyrazolyl rings, with average values of $35.58^{\circ}$ $\left(\mathbf{1}_{\mathrm{HS}}\right)$ and $51.26^{\circ}\left(\mathbf{1}_{\mathrm{LS}}\right)$. The theoretical analysis of the energy of a phenylpyrazole moiety as a function of the torsion angle unveils a significant destabilization of the system as it is removed from planarity by rotation about the CC bond 


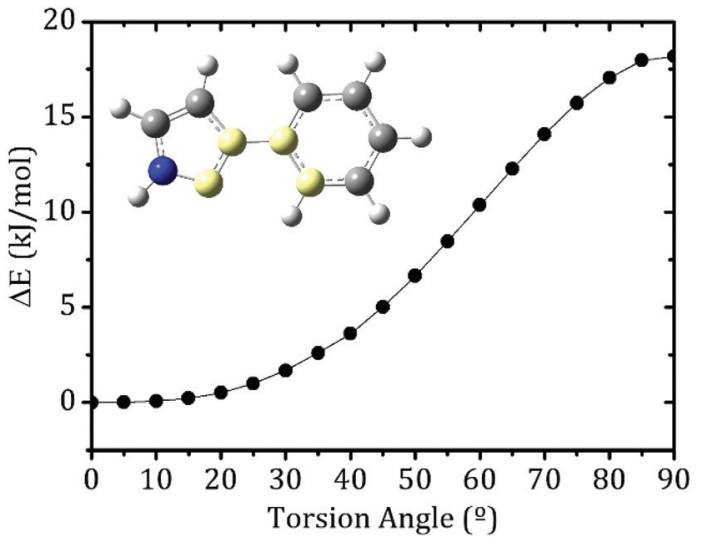

Fig. 3 Computed energy variation of a phenylpyrazole moiety as a function of the torsion angle between both aromatic rings, as gauged by the torsion angle featured by the four atoms highlighted in yellow in the inset.

between both rings (Fig. 3), which indicates that this factor would favour the HS state. From this calculation, a change from $35^{\circ}$ to $50^{\circ}$, as observed in the optimized structures, corresponds to a loss of $c a .4 \mathrm{~kJ} \mathrm{~mol}^{-1}$.

Considering that 1 contains two such units, the effective stabilization of $\mathbf{1}_{\mathrm{HS}}$ on this account would be ca. $8 \mathrm{~kJ} \mathrm{~mol}^{-1}$, close to the calculated $c a .12 \mathrm{~kJ} \mathrm{~mol}^{-1} \mathrm{HS} v s$. LS energy difference related to the Ph ring. The reason for an increased planarity loss upon HS to LS transition is the added steric hindrance between the ligands generated with the compression of the $\mathrm{FeN}_{6}$ core (Fig. S8†). The absence of this additional rotation would force, in the LS state, the presence of a $2.3 \AA \mathrm{C} \cdots \mathrm{H}$ contact, in addition to other $\mathrm{C} \cdots \mathrm{C}, \mathrm{C} \cdots \mathrm{H}$ or $\mathrm{H} \cdots \mathrm{H}$ contacts near $2.5 \AA$, which is extremely unfavorable energetically. This analysis clearly indicates that the reason why the HS state is blocked for compounds $\mathbf{1 a}$ and $\mathbf{1 b}$ is the energy penalty that would signify the additional rotation of the $\mathrm{Ph}$ group upon SCO, as the only mechanism to avoid intramolecular, interligand steric hindrance. It must be mentioned that the torsion angles observed in $\mathbf{1 a}$ and $\mathbf{1} \mathbf{b}$ are larger than the angles predicted by the calculations for $\mathbf{1}_{\mathrm{HS}}$, which would bring the former structures closer to the calculated $\mathbf{1}_{\mathrm{LS}}$ one than the latter. Comparing $\mathbf{1 a}$ and $\mathbf{1 b}$ with $\mathbf{1}_{\mathrm{LS}}$ would decrease the size of the "torque" energy that causes the trapping of the HS state. However, in the absence of any experimental LS structure, using both computed configurations to estimate the change in torsion that would occur upon SCO is the most reasonable option.

In light of the above results, it becomes important to identify in the literature other possible examples affected by the same effect. Indeed, two $\mathrm{Fe}(\mathrm{II}) / \mathrm{R}-1 \mathrm{bpp}$ complexes $(\mathrm{R}-1 \mathrm{bpp}=$ 2,6-bis-(pyrazol-1-yl)-pyridine with a substituent $\mathrm{R}$ in the position 3 of each pyrazole; $\left.\mathrm{R}=\mathrm{Ph}, \mathrm{CO}_{2} \mathrm{Et}\right)^{20}$ were found to exhibit the same behaviour as $\mathbf{1 a}$ and $\mathbf{1 b}$, with a HS state up to $300 \mathrm{~K}$. The species with the phenyl substituent is clearly analogous to the complex reported here. The ethoxycarbonyl group also probably requires energy to deviate from coplanarity with the pyridine ligand, thus blocking the system in the HS state as well. Interestingly, the related compound with $\mathrm{R}=$ mesityl $^{29}$ shows the opposite behaviour and it is found in the LS state. Here, the aromatic substituent of pyrazole is perpendicular to the latter, forced by the steric hindrance of the methyl groups, thus deactivating the blocking of the HS state. These two behaviours are therefore consistent with the findings reported here.

In summary, the ligand Ph1,3bpp, featuring a Ph substituent at the 1,3bpp core, was prepared in order to show that the SCO of an Fe(II) chromophore could be blocked by only intramolecular forces, with no effect from the crystal field. The new compound $\left[\mathrm{Fe}(\mathrm{Ph} 1,3 \mathrm{bpp})_{2}\right]\left(\mathrm{ClO}_{4}\right)_{2} \cdot \mathrm{C}_{3} \mathrm{H}_{6} \mathrm{O}$ (1a) undergoes a SCSC complete exchange of lattice acetone molecules by $\mathrm{H}_{2} \mathrm{O}$ in the atmosphere, yielding $\left[\mathrm{Fe}(\mathrm{Ph} 1,3 \mathrm{bpp})_{2}\right]\left(\mathrm{ClO}_{4}\right)_{2} \cdot 0.5 \mathrm{H}_{2} \mathrm{O}$ (1b). Both solvatomorphs retain the HS state down to $5 \mathrm{~K}$, unlike the compound $\left[\mathrm{Fe}(1,3 \mathrm{bpp})_{2}\right]\left(\mathrm{ClO}_{4}\right)_{2}$ (2), prepared with the parent ligand. Theoretical calculations of $\left[\mathrm{Fe}(\mathrm{Ph} 1,3 \mathrm{bpp})_{2}\right]^{2+}$ in the gas phase show that indeed its optimized structure in the LS state is less stable than in the HS state because the Ph group needs to rotate to alleviate the steric hindrance caused by the neighboring ligand. The rotation implies an energetic penalty associated with the loss of planarity in the phenylpyrazol-3-yl group. This would provide an elegant rationalization of the SCO inhibition, which here would be thermodynamic and not kinetic, as rationalized theoretically ${ }^{17}$ for systems where the blocking is based on intermolecular interactions.

The authors thank the Generalitat de Catalunya for the prize ICREA Academia 2008 and 2013, the ERC for Starting Grant StG-2010-258060 (GA), MINECO for grants MAT201453961-R (OR), MAT2015-70868-ERC (OR), CTQ2012-32247 (GA) and CTQ2015-68370-P (GA, CBM), LabEx-Chemistry of Complex Systems for post-doctoral grant ANR-10LABX-0026CSC (SV) and the regional High-Performance Computing (HPC) center in Strasbourg for computational resources (SV).

\section{Notes and references}

1 Spin-Crossover Materials: Properties and Applications, ed. M. A. Halcrow, John Wiley \& Sons Ltd, 2013.

2 P. Gamez, J. Sanchez Cósta, M. Quesada and G. Aromí, Dalton Trans., 2009, 7845-7853.

3 A. Bousseksou, G. Molnár, L. Salmon and W. Nicolazzi, Chem. Soc. Rev., 2011, 40, 3313-3335.

4 O. Kahn, J. Kröber and C. Jay, Adv. Mater., 1992, 4, 718-728.

5 K. S. Murray, Eur. J. Inorg. Chem., 2008, 3101-3121.

6 J. A. Real, A. B. Gaspar and M. C. Muñoz, Dalton Trans., 2005, 2062-2079.

7 P. Gütlich and H. A. Goodwin, Top. Curr. Chem., 2004, 233, $1-47$.

8 C. Lefter, V. Davesne, L. Salmon, G. Molnár, P. Demont, A. Rotaru and A. Bousseksou, Magnetochemistry, 2016, 2, 18. 
9 M. D. Manrique-Juarez, S. Rat, L. Salmon, G. Molnár, C. M. Quintero, L. Nicu, H. J. Shepherd and A. Bousseksou, Coord. Chem. Rev., 2016, 308, 395-408.

10 M. A. Halcrow, Chem. Lett., 2014, 43, 1178-1188.

11 J. A. Real, A. B. Gaspar, V. Niel and M. C. Muñoz, Coord. Chem. Rev., 2003, 236, 121-141.

12 M. Shatruk, H. Phan, B. A. Chrisostomo and A. Suleimenova, Coord. Chem. Rev., 2015, 289, 62-73.

13 N. Ortega-Villar, M. Muñoz and J. Real, Magnetochemistry, 2016, 2, 16.

14 G. A. Craig, J. Sánchez Costa, O. Roubeau, S. J. Teat and G. Aromí, Chem. - Eur. J., 2011, 17, 3120-3127.

15 M. A. Halcrow, Chem. Soc. Rev., 2011, 40, 4119-4142.

16 G. A. Craig, J. Sanchez Costa, O. Roubeau, S. J. Teat and G. Aromí, Chem. - Eur. J., 2012, 18, 1170311715.

17 S. Vela, J. J. Novoa and J. Ribas-Ariño, Phys. Chem. Chem. Phys., 2014, 16, 27012-27024.

18 A. Santoro, L. J. K. Cook, R. Kulmaczewski, S. A. Barrett, O. Cespedes and M. A. Halcrow, Inorg. Chem., 2015, 54, 682-693.

19 S. Vela, C. Gourlaouen, M. Fumanal and J. Ribas-Ariño, Magnetochemistry, 2016, 2, 6.
20 J. Elhaik, D. J. Evans, C. A. Kilner and M. A. Halcrow, Dalton Trans., 2005, 1693-1700.

21 M. A. Halcrow, Coord. Chem. Rev., 2009, 253, 2493-2514.

22 G. A. Craig, O. Roubeau and G. Aromí, Coord. Chem. Rev., 2014, 269, 13-31.

23 L. A. Barrios, C. Bartual-Murgui, E. Peyrecave-Lleixà, B. Le Guennic, S. J. Teat, O. Roubeau and G. Aromí, Inorg. Chem., 2016, 55, 4110-4116.

24 D. Gentili, N. Demitri, B. Schafer, F. Liscio, I. Bergenti, G. Ruani, M. Ruben and M. Cavallini, J. Mater. Chem. C, 2015, 3, 7836-7844.

25 L. J. K. Cook, R. Kulmaczewski, O. Cespedes and M. A. Halcrow, Chem. - Eur. J., 2016, 22, 1789-1799.

26 J. Sánchez Costa, S. Rodríguez-Jimenez, G. A. Craig, B. Barth, C. M. Beavers, S. J. Teat and G. Aromí, J. Am. Chem. Soc., 2014, 136, 3869-3874.

27 G. Aromí, C. M. Beavers, J. Sánchez Costa, G. A. Craig, G. Mínguez Espallargas, A. Orera and O. Roubeau, Chem. Sci., 2016, 7, 2907-2915.

28 C. Bartual-Murgui, C. Codina, O. Roubeau and G. Aromí, Chem. - Eur. J., 2016, 22, 12767-12776.

29 J. M. Holland, S. A. Barrett, C. A. Kilner and M. A. Halcrow, Inorg. Chem. Commun., 2002, 5, 328-332. 